\title{
Evaluation of the effect of various main elements on the PrPsc detection by real-time quaking-induced conversion assay
}

\author{
KANG XIAO $^{1,2^{*}}$, QI SHI ${ }^{1,2^{*}}$, WEI ZHOU ${ }^{1,2}, \mathrm{CHEN} \mathrm{GAO}^{1,2}, \mathrm{CAO} \mathrm{CHEN}^{1,2}$, \\ BAO-YUN ZHANG ${ }^{1,2}$, JING WANG $^{1,2}$ and XIAO-PING DONG ${ }^{1,2}$

\begin{abstract}
${ }^{1}$ State Key Laboratory for Infectious Disease Prevention and Control, Collaborative Innovation Center for Diagnosis and Treatment of Infectious Diseases (Zhejiang University), Hangzhou, Zhejiang $310003 ;{ }^{2}$ National Institute for Viral Disease Control and Prevention, Chinese Center for Disease Control and Prevention, Beijing 102206, P.R. China
\end{abstract}

Received September 4, 2017; Accepted July 4, 2018

DOI: $10.3892 / \mathrm{ijmm} .2018 .3867$

\begin{abstract}
In order to definitively diagnosis sporadic Creutzfeldt-Jakob disease (sCJD), brain tissue is currently required. Therefore, there is a great need for tests that can detect SCJD in body fluids or other types of tissues. Different variables, including the amount of recombinant celluar prion protein $\left(\mathrm{rrr}^{\mathrm{C}}\right)$, salt, cleaning surfactants and thioflavin $\mathrm{T}$ (ThT), in human cerebrospinal fluid (CSF) were evaluated. The reagent concentrations of $1 \mathrm{X}$ PBS, $170 \mathrm{mM} \mathrm{NaCl}, 1 \mathrm{mM}$ EDTA, $0.01 \mathrm{mM}$ ThT and $0.001 \%$ SDS, and the amounts of $10 \mu \mathrm{g} \mathrm{rPrP}$ and $10 \mu \mathrm{l} \mathrm{CSF}$ were considered to be optimal for the real-time quaking-induced conversion (RT-QuIC) assay. Using these conditions, the RT-QuIC assay for prion protein $\left(\mathrm{PrP}^{\mathrm{Sc}}\right)$ detection was observed to be sensitive to $10^{-8}$ diluted brain homogenates of hamsters infected with the $263 \mathrm{~K}$ scrapie strain. Furthermore, CSF samples from 70 probable sCJD cases and 48 non-CJD cases were preliminarily screened. A substantial proportion of sCJD samples $(57.14 \%)$ tested positive by RT-QuIC, with a short lag phase ( $<50 \mathrm{~h}$ post-reaction) and high peak ThT values $(>25,000$ relative fluorescence units). By contrast, only a small number of non-CJD samples displayed weakly positive results, and these were detected at a later stage ( $>50 \mathrm{~h}$ post-reaction) and had much lower ThT values. In conclusion, the RT-QuIC assay in CSF samples reported in the present study may provide a useful pre-mortem tool for the diagnosis of SCJD, particularly in China where postmortem examination is rarely conducted.
\end{abstract}

Correspondence to: Dr Qi Shi or Dr Xiao-Ping Dong, National Institute for Viral Disease Control and Prevention, Chinese Center for Disease Control and Prevention, 155 Chang-Bai Road, Beijing 102206, P.R. China

E-mail: shiqi76@126.com

E-mail: dongxp238@sina.com

*Contributed equally

Key words: Creutzfeldt-Jakob disease, $\mathrm{PrP}^{\mathrm{Sc}}$, real-time quaking-induced conversion, cerebrospinal fluid

\section{Introduction}

Prion disease, or transmissible spongiform encephalopathy, refers to a group of fatal neurodegenerative disorders reported in humans and animals (1). Prion diseases in humans include Creutzfeldt-Jakob disease (CJD), fatal familial insomnia and Gerstmann-Sträussler-Scheinker syndrome. The most common form of human prion disease is sporadic CJD (SCJD), with a worldwide incidence of $\sim 1$ case per million individuals annually $(2,3)$. Typically, sCJD presents with rapidly progressing ataxia, dementia and myoclonus. The clinical duration of the disease is usually $<2$ years, with the majority of patients succumbing to the disease within 6 months.

A definitive diagnosis of SCJD requires neuropathological or immunochemical detection of the prion protein $\left(\mathrm{PrP}^{\mathrm{Sc}}\right)$ (4). $\mathrm{PrP}^{\mathrm{Sc}}$ is partially protease-resistant and can induce its normal cellular isoform $\left(\operatorname{PrP}^{\mathrm{C}}\right)$, to undergo a conformational change. This occurs in a self-propagating manner through a seeded aggregation process, resulting in accumulation of $\mathrm{PrP}^{\mathrm{Sc}}$ throughout the brain tissues, together with accompanying spongiform alterations, neuronal loss and gliosis $(1,5,6)$. Other than brain biopsy, there is currently no other disease-specific pre-mortem diagnostic test for sCJD (7). Diagnosis of probable sCJD is based on abnormal findings as determined by clinical examinations and laboratory tests. Abnormal test results include periodic sharp wave complexes on electroencephalogram, and altered signals on brain magnetic resonance image and/or positive detection of 14-3-3 protein in the cerebrospinal fluid (CSF) (8). Postmortem examinations are rarely performed in China due to cultural traditions (9). Therefore, methods that permit the accurate diagnosis of SCJD are required.

Numerous studies have attempted to identify biomarkers in CSF samples for the diagnosis of human prion diseases. Proteins that have showed diagnostic values include 14-3-3, tau, S100 and neuron specific enolase (10-13). However, only a positive test result for 14-3-3 protein in the CSF using western blotting is included in the diagnostic criteria for $\operatorname{sCJD}(14,15)$. The detection of $\mathrm{PrP}^{\mathrm{Sc}}$ in the CSF of patients with SCJD and other types of human prion diseases is difficult with routine testing methods, even using the sensitive protein-misfolding cyclic amplification (PMCA) technique. 
Recently, a novel technique known as real-time quaking-induced conversion (RT-QuIC) has been developed that is based on amyloid fibril formation, a characteristic feature of prion proteins (16-18). A number of studies have identified that RT-QuIC has good sensitivity and specificity for the diagnosis of sCJD using human CSF samples $(16,17)$. In the present study, a number of experimental variables that may influence RT-QuIC assay were evaluated. Using the identified optimal conditions, the capacity of the RT-QuIC assay to detect $\mathrm{PrP}^{\mathrm{Sc}}$ in the brain homogenates of $263 \mathrm{~K}$-infected hamsters and in the CSF samples of probable sCJD patients was also evaluated.

\section{Materials and methods}

Ethics statement. The present study was approved by the Ethical Committee of the National Institute for Viral Disease Control and Prevention (Beijing, China) under the protocol 2009ZX10004-101, including the use of brain samples of hamsters infected with the scrapie strain $263 \mathrm{~K}$ and CSF samples from probable sCJD patients and non-CJD patients. Informed consent was obtained from all patients prior to participation.

Expression and purification of the hamster recombinant $\operatorname{PrP}(r P r P)$ protein. Hamster $\operatorname{rrP}^{\mathrm{C}}$ protein (residues 23-231; GenBank accession no. K02234) was prepared according to the method described by Wilham et al (18) with a few modifications. Briefly, the DNA sequence of hamster $\mathrm{PrP}^{\mathrm{C}}$ was ligated into the pRSET vector (cat. no. V35120; Thermo Fisher Scientific, Inc., Waltham, MA, USA), and then the recombinant plasmid was transformed into BL21 (DE3)pLysS competent cells (cat. no. C1500; Beijing Solarbio Science \& Technology Co., Ltd., Beijing, China). We expressed $\mathrm{rPrP}^{\mathrm{C}}$ in 1 liter terrific broth medium (cat. no. 71491; Novagen; Merck KGaA, Darmstadt, Germany). Next, $\operatorname{PrP}^{\mathrm{C}}$ protein was denatured with guanidine-HCL and purified by chromatography using Ni-NTA Superflow resin (cat. no. 30430; Qiagen, Hilden, Germany) in an XK 16/40 column (GE Healthcare Life Sciences, Little Chalfont, UK) at a flow rate of $1.9 \mathrm{ml} / \mathrm{min}$. The concentration of $\operatorname{rrr}^{\mathrm{C}}$ was adjusted to $\sim 500 \mu \mathrm{g} / \mathrm{ml}$ as determined by the bicinchoninic acid reagent (cat. no. 71285; EMD Millipore, Billerica, MA, USA). The purity of the prepared $\mathrm{rPrP}^{\mathrm{C}}$ was evaluated by $15 \%$ SDS-PAGE and western blot analysis. Briefly, proteins were transferred onto a nitrocellulose filter membrane (cat. no. 10600001; GE Healthcare Life Sciences), which was then blocked with 5\% skim milk in tris-buffered saline with $0.1 \%$ Tween-20 containing $0.1 \%$ Tween-20 for $1 \mathrm{~h}$ followed by incubation with the primary antibodies against 3F4 (cat. no. MAB1562-K; EMD Millipore) and $\beta$-actin (HX1827, Beijing Huaxing Bochuang Gene Technology, Co., Ltd., Beijing, China) overnight at $4^{\circ} \mathrm{C}$. Subsequently, a horseradish peroxidase-conjugated goat anti-mouse IgG secondary antibody (cat. no. 31430; Thermo Fisher Scientific, Inc.) was incubated at $37^{\circ} \mathrm{C}$ for $2 \mathrm{~h}$. Both primary and secondary antibodies were usedat a dilution of 1:5,000. The results were scanned with Bio-Rad Molecular Imager (Bio-Rad Laboratories, Inc., Hercules, California, USA) and analyzed by ImageJ software (National Institutes of Health, Bethesda, MD, USA).
Preparation of brain homogenate $(\mathrm{BH})$ samples. A total of 10 3-week-old female Syrian golden hamsters $(45.8 \pm 1.3 \mathrm{~g}$; cat. no. 501; Beijing Vital River Laboratory Animal Technology Co., Ltd., Beijing, China) were intracerebrally inoculated with $5 \mu \mathrm{l}$ hamster-adapted scrapie agents $263 \mathrm{~K}(19,20)$. Animals were housed withfree access to food and water under a humidity between $40-70 \%$, temperature between $20-26^{\circ} \mathrm{C}$ and a $12 \mathrm{~h}$ light/dark cycle. The incubation time of the $263 \mathrm{~K}$-infected hamsters was $70.5 \pm 4.93$ days $(21,22)$. Animals were sacrificed using ether and exsanguinated, and then the brains were surgically removed from each hamster. Subsequently, 10\% BHs were prepared in lysis buffer $(100 \mathrm{mM} \mathrm{NaCl}, 10 \mathrm{mM}$ EDTA, $0.5 \%$ Nonidet P-40, $0.5 \%$ sodium deoxycholate, $10 \mathrm{mM}$ Tris, $\mathrm{pH} 7.5)$ according to a previously described protocol $(21,22)$. In order to set up a $\mathrm{PrP}^{\mathrm{Sc}}$ panel for RT-QuIC, serially diluted (from $10^{-3}$ to $10^{-9}$ ) Sc-BH samples were prepared. Proteinase K-resistant PrP signals were detectable in Sc-BH samples of $10^{-3}$ using routine western blotting.

CSF samples. A total of $70 \mathrm{CSF}$ samples from probable sCJD (63.3 \pm 8.8 years old, male/female ratio: 1.12$)$ and $48 \mathrm{CSF}$ samples from non-CJD patients $(55.1 \pm 14.5$ years old, male/female ratio: 1.28) were included in the current study. Informed consent was obtained from all patients prior to participation. All the samples were collected at the CJD surveillance network of hospitals without any additional treatments and sent to the CJD Surveillance Center during Jan to Dec in 2015. The diagnosis of probable sCJD was conducted according to the diagnostic criteria for CJD issued by the World Health Organization and the surveillance document for CJD issued by Chinese Center for Disease Control and Prevention (Beijing, China) (9). Non-CJD cases included patients whose clinical manifestations and examinations did not fulfill the diagnostic criteria for human prion disease even after follow-up, or patients who had other diagnoses. The samples were collected from the CSF Bank in the China CJD Surveillance Center attached to China CDC (Beijing, China), and $200 \mu \mathrm{l}$ from each CSF sample was obtained. All enrolled CSF samples were free of blood contamination. Routine CSF biochemistry assays of those specimens, including cell count, glucose level and total protein, were all within the normal ranges.

Evaluation of the effect of various main elements on the reactivity of $R T-Q u I C$. To identify any factors that affect the RT-QuIC assay for detecting $\mathrm{PrP}^{\mathrm{Sc}}$, a set of variables were independently evaluated. The basic elements in the working buffer were $1 \mathrm{X}$ PBS, 85-340 mM NaCl, 0.5-2 mM EDTA, 10-80 $\mu \mathrm{M}$ thioflavin $\mathrm{T}$ (ThT) and 5-20 $\mu \mathrm{g} \mathrm{rPrP}^{\mathrm{C}}$. These concentrations were selected based on published data and our previous experiments $(16,23)$. In addition, $10^{-5}$ diluted $\mathrm{BH}$ from $263 \mathrm{~K}$-infected hamster at the terminal stage (Sc-BH) and $\mathrm{BH}$ from age-matched normal hamsters (Nor-BH) were also prepared as the positive and negative controls, respectively.

RT-QuIC assay. RT-QuIC was conducted in a black 96-well, optical-bottomed plate (Nunc 265301; Thermo Fisher Scientific, Inc.) on a BMG FLUOstar microplate reader (BMG LABTECH GmbH, Ortenberg, Germany). Next, $1 \mu 1$ diluted $\mathrm{BH}$ or 5-30 $\mu \mathrm{l}$ CSF sample was mixed with $1 \mathrm{X}$ PBS, 
$170 \mathrm{mM} \mathrm{NaCl}, 1 \mathrm{mM}$ EDTA, $0.01 \mathrm{mM}$ ThT, $0.001 \%$ SDS and $10 \mu \mathrm{g} \mathrm{rPrPC}$ in a final volume of $100 \mu 1$. Each sample was assayed in triplicated. Each reaction contained the following control groups: Blank (reaction buffer only), positive (Sc-BH) and negative (Nor-BH). The working conditions were as follow: Temperature, $50^{\circ} \mathrm{C}$; vibration speed, $1.996 \mathrm{x} \mathrm{g}$; vibration/incubation time, 90/30 sec; total reaction time, $90 \mathrm{~h}$. ThT fluorescence (excitation wavelength, $450 \mathrm{~nm}$; emission wavelength, $480 \mathrm{~nm}$ ) was automatically measured every $30 \mathrm{~min}$ and expressed as relative fluorescence units (rfu). The cutoff value was set as the mean value of the negative controls plus 3 times the standard deviation. A sample was considered to be positive when $\geq 2$ wells revealed positive reaction curves.

\section{Results}

Determination of appropriate $r P r P^{C}$ concentration. Different amounts $(5,10,15$ and $20 \mu \mathrm{g})$ of purified $\mathrm{rPrP}^{\mathrm{C}}$, serving as the substrate, were subjected to RT-QuIC. Using Sc-BH as the seed, which induces the fibrillation of $\operatorname{rPrP}^{\mathrm{C}}$, increases in the ThT fluorescence curves were observed in the reactions of 10,15 and $20 \mu \mathrm{g} \mathrm{rPrP}$, which began to increase at $\sim 30 \mathrm{~h}$ post-reaction, while only a slight elevation of the ThT value was detected in the $5 \mu \mathrm{g} \mathrm{PrP}^{\mathrm{C}}$ reaction (Fig. 1). However, a positive increase of ThT value was also recorded in part of duplication of $20 \mu \mathrm{g} \mathrm{rPrP}^{\mathrm{C}}$ using Nor-BH as the seed, indicating a false positive reaction. Based on these data, $10 \mu \mathrm{g}$ $\mathrm{rPrP}^{\mathrm{C}}$ was suggested to be the optimal working amount as the substrate in RT-QuIC.

Determination of appropriate NaCl concentration. Different concentrations of $\mathrm{NaCl}(0,85,170,255$ and $340 \mathrm{mM})$ were examined. In the condition of $10^{-5}$ dilutedSc-BH, four preparations containing $\mathrm{NaCl}$ demonstrated positive reaction. The RT-QuIC reactivity was revealed to be $\mathrm{NaCl}$ dose-dependent. Along with the increase in the amount of $\mathrm{NaCl}$, the positive conversion time post-reaction was decreased and the peak values of ThT values increased. However, increased ThT curves were also observed in the preparations of negative control containing 255 and $340 \mathrm{mM} \mathrm{NaCl}$. This suggested that a high concentration of $\mathrm{NaCl}$ was able to increase the reactivity of RT-QuIC, as well as increase the possibility of false positive (Fig. 2). Thus, the $\mathrm{NaCl}$ concentration of $170 \mathrm{mM}$ was selected for subsequent experiments.

Determination of appropriate EDTA concentration. Different concentration of EDTA $(0,0.5,1,1.5$ and $2 \mathrm{mM})$ were also tested in the RT-QuIC assay containing $10^{-5}$ diluted Sc-BH. After $34 \mathrm{~h}$, the reaction curve with $1 \mathrm{mM}$ EDTA began to increase, followed by the curves of the 1.5 and $2 \mathrm{mM}$ EDTA reactions. At a relatively late stage, the reactions with 0 and $0.5 \mathrm{mM}$ EDTA exhibited weak positive results. These data illustrated that certain concentrations of EDTA (particularly $1 \mathrm{mM}$ ) help the reactivity of RT-QuIC assay, whereas high concentrations of EDTA inhibit the assay (Fig. 3).

Determination of appropriate ThT final concentration. Four different final concentrations of ThT $(10,20,40$ and $80 \mu \mathrm{M})$ were also examined in the RT-QuIC assay containing $10^{-5}$ diluted

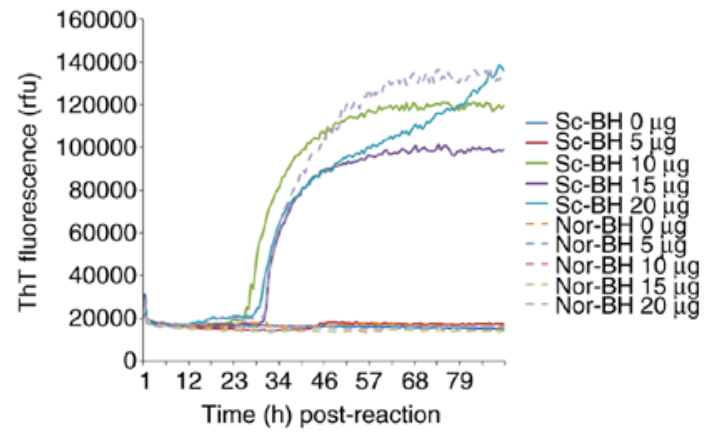

Figure 1. Effects of different $\mathrm{rPrP}^{\mathrm{C}}$ concentrations on the detection capacity of real-time quaking-induced conversion assay. Different amounts of $\mathrm{rPrP}^{\mathrm{C}}$ $(0,5,10,15$ and $20 \mu \mathrm{g})$ were added into the samples containing $10^{-5}$ diluted BH from $263 \mathrm{~K}$ hamsters or normal controls. ThT, thioflavin T; rfu, relative fluorescence units; $\mathrm{rPrP}^{\mathrm{C}}$, recombinant cellular prion protein; $\mathrm{Sc}-\mathrm{BH}$, $\mathrm{BH}$ from $263 \mathrm{~K}$-infected hamster at the terminal stage; Nor- $\mathrm{BH}, \mathrm{BH}$ from age-matched normal hamsters; $\mathrm{BH}$, brain homogenate.

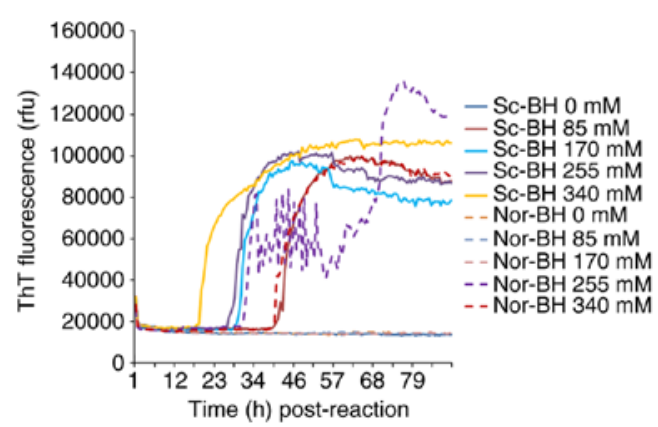

Figure 2. Effects of different concentrations of $\mathrm{NaCl}$ on real-time quaking-induced conversion assay. Different concentrations $(0,85$, 170,255 and $340 \mathrm{mM}$ ) of $\mathrm{NaCl}$ were added into samples containing $10^{-5}$ times diluted $\mathrm{BH}$ from $263 \mathrm{~K}$ hamsters or normal controls. ThT, thioflavin T; rfu, relative fluorescence units; $\mathrm{Sc}-\mathrm{BH}, \mathrm{BH}$ from $263 \mathrm{~K}$-infected hamster at the terminal stage; Nor-BH, $\mathrm{BH}$ from age-matched normal hamsters; $\mathrm{BH}$, brain homogenate.

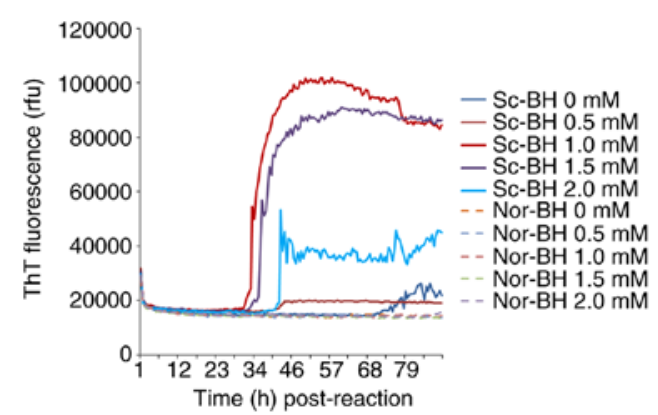

Figure 3. Effects of different concentrations of EDTA on real-time quaking-induced conversion assay. Different concentrations $(0,0.5,1,1.5$ and $2 \mathrm{mM}$ ) of EDTA were added into samples containing $10^{-5}$ diluted $\mathrm{BH}$ from $263 \mathrm{~K}$ hamsters or normal controls. ThT, thioflavin T; rfu, relative fluorescence units; Sc-BH, BH from 263K-infected hamster at the terminal stage; Nor-BH, BH from age-matched normal hamsters; $\mathrm{BH}$, brain homogenate.

Sc-BH. All four preparations of $\operatorname{PrP}^{\mathrm{Sc}}$ exhibited positive curves at approximately $9-12 \mathrm{~h}$ post-reaction, and reached similarly high ThT fluorescence values after $30 \mathrm{~h}$ of reaction (Fig. 4). However, increased curves were also observed in the negative controls containing 20,40 and $80 \mu \mathrm{M}$ ThT, although these 


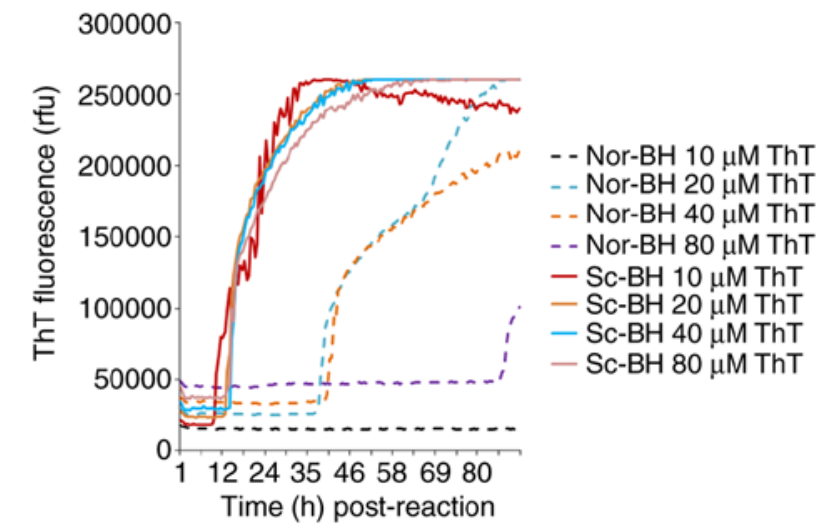

Figure 4. Effects of different concentrations of ThT on real-time quaking-induced conversion assay. Different concentrations (10, 20, 40 and $80 \mu \mathrm{M}$ ) of ThT were added into the samples containing $10^{-5}$ diluted $\mathrm{BH}$ from $263 \mathrm{~K}$ hamsters or normal controls. ThT, thioflavin T; rfu, relative fluorescence units; Sc-BH, BH from $263 \mathrm{~K}$-infected hamster at the terminal stage; Nor-BH, BH from age-matched normal hamsters; $\mathrm{BH}$, brain homogenate.

emerged with relatively long lag phases (Fig. 4). These findings suggest that an increase in ThT concentration does not enhance the sensitivity of RT-QuIC assay and provides false positive results.

Determination of appropriate SDS concentration. Different concentrations of SDS were added to reactions containing $10^{-5}$ diluted Sc-BH, resulting in final concentrations of SDS between 0.1 and $0.00001 \%$. As shown in Fig. 5A, positive reaction curves were observed in the preparations of 0.001 , 0.0001 and $0.00001 \%$ SDS, among which the reaction using $0.001 \%$ SDS exhibited a markedly shorter lag phase and higher ThT value. Positive reactivities were markedly inhibited in the presences of 0.1 and $0.01 \%$ SDS, and a false weak positive reaction was observed in the preparation of Nor-BH containing $0.01 \%$ of SDS. Furthermore, the effect of $0.001 \% \mathrm{SDS}$ on the reactivity of different amounts of $\mathrm{PrP}^{\mathrm{Sc}}$ was evaluated. In the conditions of $263 \mathrm{~K} \mathrm{BH}$ diluted for $10^{-5}$ and $10^{-7}$ times, the presence of $0.001 \%$ SDS caused a evidently quicker increase and higher ThT fluorescence values (Fig. 5B).

Determination of appropriate CSF amount. To test the potential influence of human CSF on the reactivity in RT-QuIC assays, $10^{-5}$ times diluted Sc-BH was added into $30 \mu \mathrm{l} \mathrm{CSF}$ collected from a non-CJD patient with major indexes in the CSF biochemistry within the normal range. Compared with the same dilution of Sc-BH only, the lag time and fluorescence peak in the reaction of Sc-BH in CSF were evidently longer and lower, respectively (Fig. 6A). Furthermore, $10^{-5}$ times diluted Sc-BH was separately mixed with 5, 10 and $30 \mu \mathrm{l} \mathrm{CSF}$ samples. The RT-QuIC assay results demonstrated that the reactions with 5 and $10 \mu \mathrm{l}$ CSF displayed similar positive reactivities with a much shorter lag phase and higher fluorescence peak in comparison with that of $30 \mu \mathrm{l} \mathrm{CSF} \mathrm{(Fig.} \mathrm{6B).} \mathrm{Thus,}$ it appears that certain unknown components of human CSF may hamper the RT-QuIC assay; therefore, using relatively large amount of tested CSF in RT-QuIC does not benefit the detecting ability for $\mathrm{PrP}^{\mathrm{Sc}}$.
Final experimental conditions. Based on the aforementioned data, the experimental conditions of RT-QuIC were set-up, and these involved the final concentrations of $1 \mathrm{X}$ PBS, $170 \mathrm{mM}$ $\mathrm{NaCl}, 1 \mathrm{mM}$ EDTA, $0.01 \mathrm{mM}$ ThT and $0.001 \%$ SDS with $10 \mu \mathrm{g} \mathrm{rPrP}$ and $10 \mu \mathrm{l} \mathrm{CSF}$ in a total reaction volume of $100 \mu \mathrm{l}$. The positive control was $10^{-5}$ diluted $\mathrm{BH}$ collected from 263K-infected hamsters and the negative control was the same dilution of $\mathrm{BH}$ obtained from normal hamsters.

Evaluation of the capacity of the optimized RT-QuIC assay in the detection of $\mathrm{PrP}^{S c}$ in brain tissues and CSF samples. The serially diluted Sc-BHs $\left(10^{-3}\right.$ to $\left.10^{-9}\right)$ were separately subjected to the optimized RT-QuIC reactions. As shown in Fig. 7, the ThT fluorescence values of the preparations of blank control and Nor-BH were almost unchanged at $90 \mathrm{~h}$ post-reaction. By contrast, the ThT values of the reactions containing relatively high amounts of Sc-BH (between $10^{-3}$ and $10^{-6}$ ) increased markedly after lag phases of approximately $9-12 \mathrm{~h}$ post-reaction. The preparations of $10^{-7}$ and $10^{-8}$ diluted $\mathrm{PrP}^{\mathrm{Sc}} \mathrm{BHs}$ exhibited increased ThT values at 37 and $52 \mathrm{~h}$ post-reaction, respectively. No positive result was observed in the reaction of $10^{-9}$ diluted $\mathrm{PrP}^{\mathrm{Sc}} \mathrm{BH}$. Furthermore, the ThT fluorescence in the preparations containing high amounts of $\mathrm{PrP}^{\mathrm{Sc}}$ was evidently higher in comparison with that of reactions containing low amounts of $\mathrm{PrP}^{\mathrm{Sc}}$. The $\mathrm{PrP}^{\mathrm{Sc}}$ detection threshold of the RT-QuIC assay reported in the present study was observed to be $10^{-8}$ diluted $\mathrm{BH}$ of $263 \mathrm{~K}$-infected hamsters.

To access the efficacy of the optimized RT-QuIC assay in human SCJD CSF samples, CSF samples from 70 patients that fulfilled the diagnostic criteria for probable sCJD and 48 patients that were classified as non-CJD were assayed. In total, 10 or $30 \mu \mathrm{l} \mathrm{CSF}$ sample from each patient was separately tested. Using $30 \mu \mathrm{l} \mathrm{CSF}, 11(15.71 \%)$ samples in the probable sCJD group tested positive, while all tested samples in the non-CJD group were negative. Positive conversion times varied between 8 and $28 \mathrm{~h}$ post-reaction, while the peak ThT value varied between 44,000 and 61,000 rfu. Using $10 \mu 1$ CSF, 40 (57.14\%) samples of probable sCJD tested positive, whereas 11 samples of non-CJD also exhibited weakly positive results. Further analysis of the positive reaction patterns in the RT-QuIC assay revealed notably different profiles between the groups of probable sCJD (28 samples) and non-CJD (11 samples). As shown in Fig. 8, the majority of the samples from the probable sCJD group tested positive within $50 \mathrm{~h}$ post-reaction (27/28 samples), and exhibited peak ThT values of $>25,000 \mathrm{rfu}$ (26/28 samples). By contrast, only 1 case of non-CJD tested positive within $50 \mathrm{~h}$ post-reaction, and the peak ThT values of all non-CJD case were $<25,000 \mathrm{rfu}$. These data indicate that applying the CSF RT-QuIC assay with the aforementioned experimental conditions was able to detect substantially more probable sCJD cases, with a significantly shorter lag phase $(<50 \mathrm{~h}$ post-reaction) and higher ThT fluorescence values $(>25,000 \mathrm{rfu})$.

\section{Discussion}

The results of RT-QuIC, which is considered to be a sensitive assay, can be affected by a variety of factors. Under a specific working temperature and vibration speed, the effect of certain variables on the RT-QuIC detection capacity was evaluated 

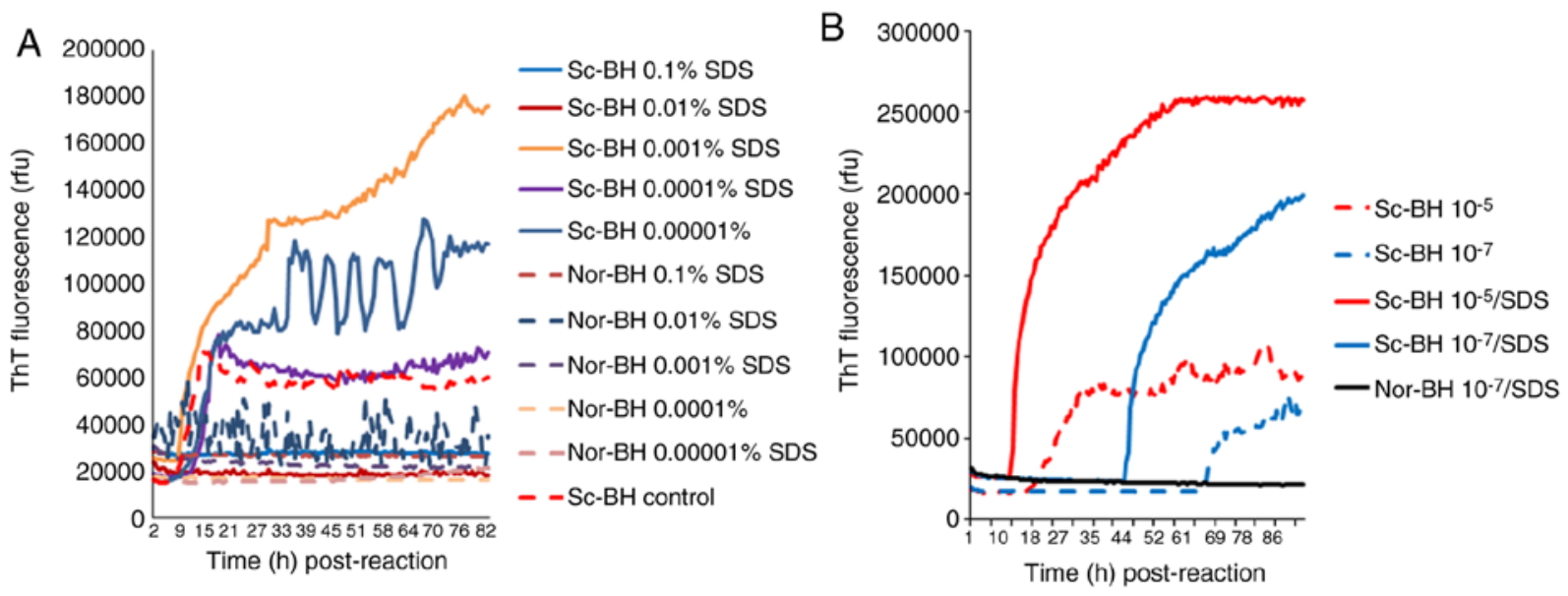

Figure 5. Effects of different concentrations of SDS on real-time quaking-induced conversion assay. (A) Assay of different SDS concentrations in samples containing a fixed amount of $\mathrm{PrP}^{\mathrm{Sc}}$. Different final concentrations $(0.1-0.00001 \%)$ of SDS were added into the tested samples containing $10^{-5}$ diluted $\mathrm{BH}$ from $263 \mathrm{~K}$ hamsters or normal controls. (B) Assay of a fixed amount of SDS in samples containing different amounts of PrP $\mathrm{P}^{\mathrm{Sc}}$. Samples containing $10^{-5}$ and $10^{-7}$ diluted $263 \mathrm{~K}$ hamster $\mathrm{BH}$ were mixed with a final concentration of $0.001 \%$ SDS. PrP ${ }^{\mathrm{Sc}}$, prion protein; ThT, thioflavin T; rfu, relative fluorescence units; Sc-BH, BH from 263K-infected hamster at the terminal stage; Nor-BH, BH from age-matched normal hamsters; BH, brain homogenate.
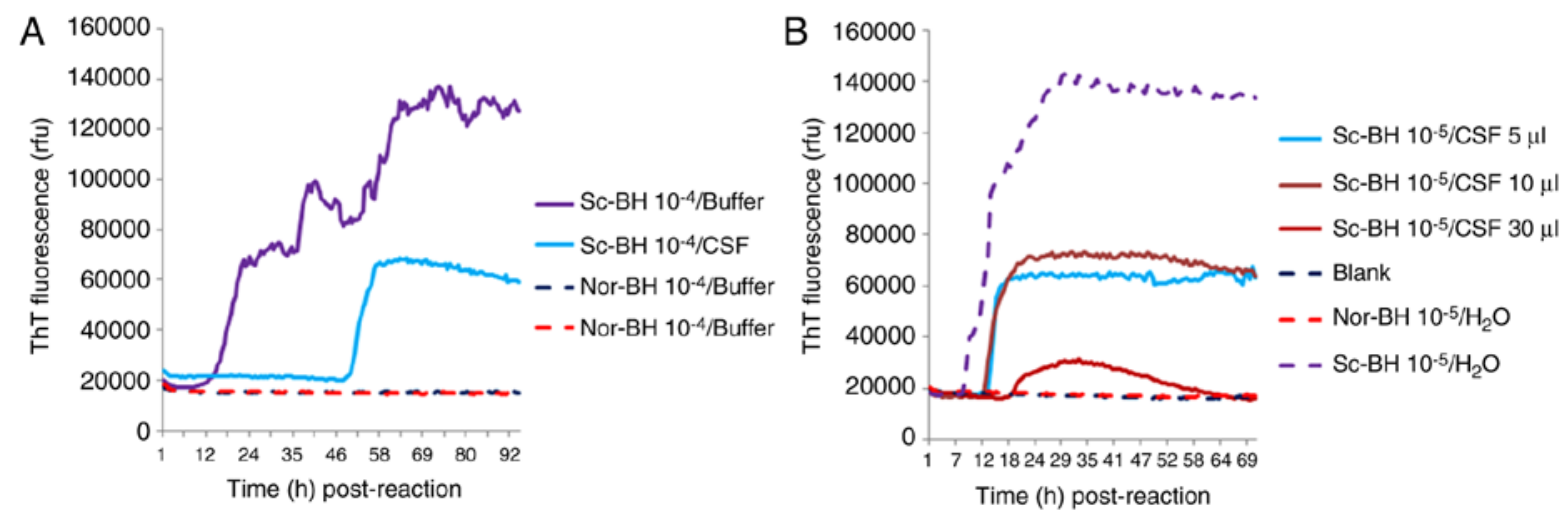

Figure 6. Effects of different volumes of CSF on real-time quaking-induced conversion assay. (A) Examination of reactions with or without human CSF. Samples containing $10^{-4}$ diluted $263 \mathrm{~K}$ hamster BH were mixed with or without $30 \mu \mathrm{l} \mathrm{CSF}$. (B) Examination of reactions with different amounts of human CSF $(5,10$ and $30 \mu \mathrm{l})$ added into the samples containing $10^{-5}$ diluted $263 \mathrm{~K}$ hamster BH. CSF, cerebrospinal fluid; ThT, thioflavin T; rfu, relative fluorescence units; Sc-BH, BH from 263K-infected hamster at the terminal stage; Nor-BH, BH from age-matched normal hamsters; BH, brain homogenate.

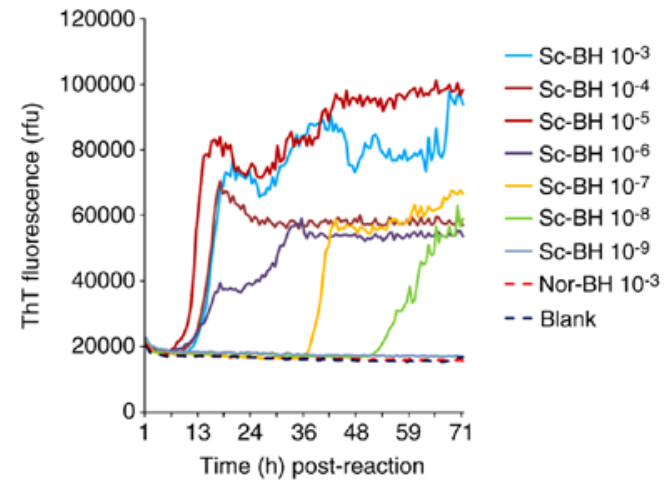

Figure 7. Sensitivity of the real-time quaking-induced conversion assay for detecting $\mathrm{PrP}^{\mathrm{Sc}}$. Different dilutions $\left(10^{-3}\right.$ to $\left.10^{-9}\right)$ of $263 \mathrm{~K}$ hamster $\mathrm{BH}$ were examined. Samples of $10^{-3}$ to $10^{-6}$ diluted $263 \mathrm{~K}$ hamster BH exhibited positive reactions after lag phases of $9-12 \mathrm{~h}$. Samples of $10^{-7}$ and $10^{-8}$ diluted $\mathrm{BH}$ displayed positive curves at 37 and $52 \mathrm{~h}$ post-reaction. No positive reaction was recorded for the sample containing $10^{-9}$ dilution, as well as those of normal hamster brain or blank control. $\mathrm{PrP}^{\mathrm{Sc}}$, prion protein; ThT, thioflavin T; rfu, relative fluorescence units; $\mathrm{Sc}-\mathrm{BH}, \mathrm{BH}$ from 263K-infected hamster at the terminal stage; Nor-BH, BH from age-matched normal hamsters; $\mathrm{BH}$, brain homogenate.

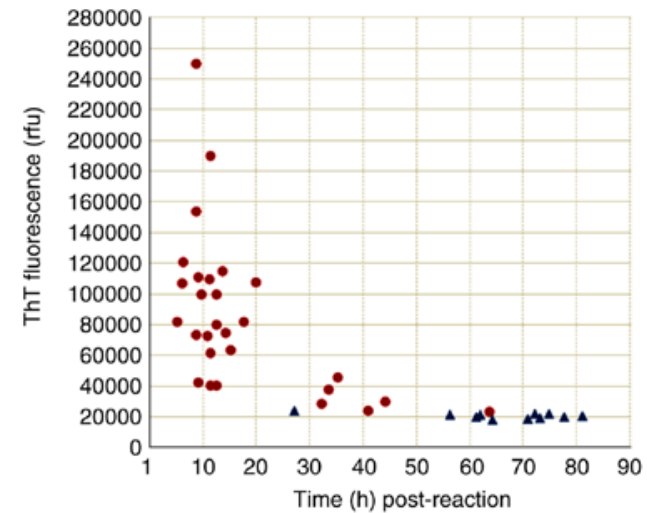

Figure 8. Dot plot of the positive CSF real-time quaking-induced conversion assay results for the probable sCJD and non-CJD groups. A total of $28 \mathrm{CSF}$ samples from probable SCJD cases (red dots) and 11 CSF samples from non-CJD cases (blue triangles) with positive results were included. Among the probable sCJD samples, 27 out of 28 exhibited positive results within $50 \mathrm{~h}$ post-reaction, while the peak ThT values of 26 cases were $>25,000 \mathrm{rfu}$. Only 1 non-CJD case was positive within $50 \mathrm{~h}$ post-reaction, and the peak ThT values of all the non-CJD cases were $<25,000 \mathrm{rfu}$. CSF, cerebrospinal fluid; CJD, Creutzfeldt-Jakob disease; ThT, thioflavin T; rfu, relative fluorescence units. 
in the present study. Similarly to a previous study (12), a type of E. coli-expressed full-length wild-type hamster rPrP was used as substrate in the RT-QuIC assay reported in the current study. In a certain range of $\mathrm{rPrP}^{\mathrm{C}}$ concentrations, the sensitivity of RT-QuIC exhibits a positive association with the amount of the input $\mathrm{rPrP}^{\mathrm{C}}$, however, false positive results are also easily inducible. PrP protein is capable of spontaneous fibrillation in vitro when incubated at certain conditions, for it was reported that agitation alone induces de novo conversion of recombinant prion proteins to b-sheet rich fibrils (24). Using PMCA, a wild-type mouse rPrP protein can be converted into a pathogenic isoform that has the biochemical characteristics of $\mathrm{PrP}^{\mathrm{Sc}}$ in vitro, as well as the typical infectivity on experimental rodents (25). Therefore, careful consideration of the $\mathrm{rPrP}^{\mathrm{C}}$ amount and of the normal negative control is important, particularly when using different batches of purified $\mathrm{rPrP}^{\mathrm{C}}$ protein.

Partially denatured $\mathrm{rPrP}^{\mathrm{C}}$ protein may help to form fibrils in RT-QuIC assay. In addition, using specific concentrations of PBS, $\mathrm{NaCl}$, EDTA and SDS in the working buffer benefits the induction of earlier and higher positive reactions in the RT-QuIC assay. Meanwhile, excessive concentrations of salt and cleaning surfactants lead to a false positive result or inhibit the reactivity of RT-QuIC. ThT is a commonly used chemical for diagnosis of the amyloid structure; however, it is not perfectly specific for amyloid (26). The spectroscopic change of ThT may differ largely, depending on the particular protein and experimental conditions. In the present study, it was also observed that a high concentration of ThT induced evidently false positive reactions in the negative control sample. Thus, a careful balance of the use of salt, cleaning surfactants and ThT concentrations in the reaction buffer is essential for ensuring the sensitivity and specificity of RT-QuIC assay.

The current study also demonstrated that the amount of human CSF used can inhibit the RT-QuIC assay. Human CSF samples with normal biochemical profiles significantly reduced the ThT fluorescence intensity and prolonged the lag time until a positive reaction was detected. These results suggested that there were certain unknown factors in human CSF that inhibited the $\mathrm{PrP}^{\mathrm{Sc}}$ amyloid formation in the RT-QuIC assay. An improved RT-QuIC assay sensitivity was detected in reactions using a relatively low amount of human CSF (5 and $10 \mu \mathrm{l}$ ) under the experimental conditions of the current study. Further studies identifying and removing any inhibitor(s) in human CSF would improve the sensitivity of CSF RT-QuIC assay for SCJD detection.

Using optimized experimental conditions in the present study, samples containing $10^{-8}$ dilution of $263 \mathrm{~K}$ hamster BHwere successfully detected, which indicated a higher $\mathrm{PrP}^{\mathrm{Sc}}$ detection capacity in comparison with that of routine western blot analysis $\left(10^{-3}\right)$ and that of PMCA $\left(10^{-5}\right)$ using $10 \% \mathrm{BH}$ of normal hamsters as a substrate (27). A number of CSF samples from probable CJD and non-CJD patients were also preliminarily screened in the present study. Approximately $60 \%$ of the CSF samples from the probable sCJD patient group tested positive. Notably, the majority of these results occurred within $50 \mathrm{~h}$ post-reaction (median, $11.85 \mathrm{~h}$ ), and had high peak ThT fluorescence values (median, 77,500 rfu). Certain CSF samples from the non-CJD patient group also exhibited weakly positive reactions, however, these had markedly longer lag phases (median, $70.70 \mathrm{~h}$ post-reaction) and evidently reduced peak ThT values (median, 21,000 rfu). On the basis of these data, it is proposed that $<50 \mathrm{~h}$ post-reaction and/or $>25,000 \mathrm{rfu}$ should be used as the cut-off values for positive test results when applying the CSF RT-QuIC assay with the experimental conditions reported in the current study. Certainly, a larger number of samplesare required to further validate the suitability of these criteria in the clinical diagnosis of sCJD.

In conclusion, the present study evaluated the factors which may affect the RT-QuIC assay, and confirmed that RT-QuIC was capable of detecting traces of $\mathrm{PrP}^{\mathrm{Sc}}$. Application of this assay using CSF samples in the present study revealed the potential use as a pre-mortem tool for the diagnosis of sCJD.

\section{Acknowledgements}

The authors would like to thank Dr Shelley Robison for English language editing.

\section{Funding}

This work was supported by Chinese National Natural Science Foundation Grants (grant nos. 81630062, 81301429 and 81572048), National Key Research and Development Plan (grant no. 2016YFC1202700) and SKLID Development Grant (grant nos. 2012SKLID102 and 2015SKLID503).

\section{Availability of data and materials}

The datasets used and/or analyzed during the current study are available from the corresponding author upon reasonable request.

\section{Authors' contributions}

KX designed the study, acquired the data and prepared the manuscript; QS prepared the manuscript; JW, CG and BYZ assisted in the RT-QuIC assay; CC performed statistical analysis; WZ and QS prepared the samples; XPD, who was the corresponding author, designed the study and revised the manuscript. All authors read and approved the final manuscript.

\section{Ethics approval and consent to participate}

The present study was approved by the Ethical Committee of the National Institute for Viral Disease Control and Prevention (Beijing, China; protocol 2009ZX10004-101). Informed consent was obtained from all patients prior to participation.

\section{Patient consent for publication}

Not applicable.

\section{Competing interests}

The authors declare that they have no conflict of interest. 


\section{References}

1. Prusiner SB: Prions. Proc Natl Acad Sci USA 95: 13363-13383, 1998.

2. Ladogana A, Puopolo M, Croes EA, Budka H, Jarius C, Collins S, Klug GM, Sutcliffe T, Giulivi A, Alperovitch A, et al: Mortality from Creutzfeldt-Jakob disease and related disorders in Europe, Australia, and Canada. Neurology 64: 1586-1591, 2005.

3. Chen $\mathrm{C}$ and Dong XP: Epidemiological characteristics of human prion diseases. Infect Dis Poverty 5: 47, 2016.

4. Budka H, Aguzzi A, Brown P, Brucher JM, Bugiani O, Gullotta F, Haltia M, Hauw JJ, Ironside JW, Jellinger K, et al: Neuropathological diagnostic criteria for Creutzfeldt-Jakob disease (CJD) and other human spongiform encephalopathies (prion diseases). Brain Pathol 5: 459-466, 1995.

5. Borchelt DR, Scott M, Taraboulos A, Stahl N and Prusiner SB: Scrapie and cellular prion proteins differ in their kinetics of synthesis and topology in cultured cells. J Cell Biol 110: 743-752, 1990.

6. Prusiner SB: Novel properties and biology of scrapie prions. Curr Top Microbiol Immunol 172: 233-257, 1991.

7. Manix M, Kalakoti P, Henry M, Thakur J, Menger R Guthikonda B and Nanda A: Creutzfeldt-Jakob disease: Updated diagnostic criteria, treatment algorithm, and the utility of brain biopsy. Neurosurg Focus 39: E2, 2015.

8. Zerr I, Kallenberg K, Summers DM, Romero C, Taratuto A, Heinemann U, Breithaupt M, Varges D, Meissner B, Ladogana A, et al: Updated clinical diagnostic criteria for sporadic Creutzfeldt-Jakob disease. Brain 132: 2659-2668, 2009.

9. Shi Q, Zhou W, Chen C, Gao C, Xiao K, Wang J, Zhang BY, Wang Y, Zhang F and Dong XP: Quality evaluation for the surveillance system of human prion diseases in China based on the data from 2010 to 2016. Prion 10: 484-491, 2016.

10. Hsich G, Kenney K, Gibbs CJ, Lee KH and Harrington MG: The 14-3-3 brain protein in cerebrospinal fluid as a marker for transmissible spongiform encephalopathies. N Engl J Med 335 924-930, 1996

11. Sanchez-Juan P, Sanchez-Valle R, Green A, Ladogana A, Cuadrado-Corrales N, Mitrová E, Stoeck K, Sklaviadis T, Kulczycki J, Hess K, et al: Influence of timing on CSF tests value for Creutzfeldt-Jakob disease diagnosis. J Neurol 254: 901-906, 2007.

12. Otto M, Wiltfang J, Cepek L, Neumann M, Mollenhauer B, SteinackerP,Ciesielczyk B, Schulz-Schaeffer W, Kretzschmar HA and Poser S: Tau protein and 14-3-3 protein in the differential diagnosis of Creutzfeldt-Jakob disease. Neurology 58: 192-197, 2002.

13. Beaudry P, Cohen P, Brandel JP, Delasnerie-Lauprêtre N, Richard S, Launay JM and Laplanche JL: 14-3-3 protein, neuron-specific enolase, and S-100 protein in cerebrospinal fluid of patients with Creutzfeldt-Jakob disease. Dement Geriatr Cogn Disord 10: 40-46, 1999

14. Collins S, Boyd A, Fletcher A, Gonzales M, McLean CA, Byron K and Masters CL: Creutzfeldt-Jakob disease: Diagnostic utility of 14-3-3 protein immunodetection in cerebrospinal fluid. J Clin Neurosci 7: 203-208, 2000.

15. Shi Q, Gao C, Zhou W, Zhang BY, Chen JM, Tian C, Jiang HY, Han J, Xiang NJ, Wang XF, et al: Surveillance for Creutzfeldt-Jakob disease in China from 2006 to 2007. BMC Public Health 8: 360, 2008.
16. McGuire LI, Peden AH, Orru CD, Wilham JM, Appleford NE, Mallinson G, Andrews M, Head MW, Caughey B, Will RG, et al: Real time quaking-induced conversion analysis of cerebrospinal fluid in sporadic Creutzfeldt-Jakob disease. Ann Neurol 72: 278-285, 2012

17. Atarashi R, Satoh K, Sano K, Fuse T, Yamaguchi N, Ishibashi D, Matsubara T, Nakagaki T, Yamanaka $\mathrm{H}$, Shirabe S, et al: Ultrasensitive human prion detection in cerebrospinal fluid by real-time quaking-induced conversion. Nat Med 17: 175-178, 2011.

18. Wilham JM, Orru CD, Bessen RA, Atarashi R, Sano K, Race B, Meade-White KD, Taubner LM, Timmes A and Caughey B: Rapid end-point quantitation of prion seeding activity with sensitivity comparable to bioassays. PLoS Pathog 6: e1001217, 2010.

19. Wang GR, Shi S, Gao C, Zhang BY, Tian C, Dong CF, Zhou RM, Li XL, Chen C and Dong XP: Alternations of tau protein and its phosphorylated profiles in the experimental hamsters infected by scrapie agents 263K and 139A. Bing Du Xue Bao 25: 202-207, 2009 (In Chinese)

20. Zhang BY, Tian C, Han J, Gao C, Shi Q, Chen JM, Jiang HY, Zhou W and Dong XP: Establishment of a stable $\operatorname{PrP}(\mathrm{Sc})$ panel from brain tissues of experimental hamsters with scrapie strain 263K. Biomed Environ Sci 22: 151-156, 2009.

21. Zhang J, Chen L, Zhang BY, Han J, Xiao XL, Tian HY, Li BL, Gao C, Gao JM, Zhou XB, et al: Comparison study on clinical and neuropathological characteristics of hamsters inoculated with scrapie strain $263 \mathrm{~K}$ in different challenging pathways. Biomed Environ Sci 17: 65-78, 2004.

22. Gao JM, Gao C, Han J, Zhou XB, Xiao XL, Zhang J, Chen L, Zhang BY, Hong T and Dong XP: Dynamic analyses of PrP and $\operatorname{PrP}(\mathrm{Sc})$ in brain tissues of golden hamsters infected with scrapie strain $263 \mathrm{~K}$ revealed various PrP forms. Biomed Environ Sci 17: 8-20, 2004.

23. Zanusso G, Monaco S, Pocchiari M and Caughey B: Advanced tests for early and accurate diagnosis of Creutzfeldt-Jakob disease. Nat Rev Neurol 12: 325-333, 2016.

24. Ladner-Keay CL, Griffith BJ and Wishart DS: Shaking alone induces de novo conversion of recombinant prion proteins to beta-sheet rich oligomers and fibrils. PLoS One 9: e98753, 2014.

25. Yuan Z, Yang L, Chen B, Zhu T, Hassan MF, Yin X, Zhou X and Zhao D: Protein misfolding cyclic amplification induces the conversion of recombinant prion protein to $\mathrm{PrP}$ oligomers causing neuronal apoptosis. J Neurochem 133: 722-729, 2015.

26. Biancalana M and Koide S: Molecular mechanism of Thioflavin-T binding to amyloid fibrils. Biochim Biophys Acta 1804: 1405-1412, 2010 .

27. Orru CD, Wilham JM, Vascellari S, Hughson AG and Caughey B: New generation QuIC assays for prion seeding activity. Prion 6: $147-152,2012$.

This work is licensed under a Creative Commons Attribution-NonCommercial-NoDerivatives 4.0 International (CC BY-NC-ND 4.0) License. 